\title{
Interessenförderung im DaF-Unterricht durch Methodenvielfalt? - eine empirische Untersuchung
}

\section{Andreja Reteli}

Numerous studies have confirmed that learner's interest plays a relatively significant role in achieving learning success. Our study examines the implementation of various teaching approaches in German lessons and the effect they have on student's interest for learning German. In the experimental group, three different topics were taught by the principles of Communicative Approach, CLIL - Content and Integrated Language Learning and CALL Computer assisted Language Learning. The control group was taught the same topics according to the principle oft Communicative Approach. The findings of this empirical study reveal that the interest for German language was significantly lower in the experimental group after the experiment. Possible reasons for lower interest are discussed and some teaching strategies are presented.

Interest - CLIL - CALL - communicative Approach - German as a foreign language

Bisherige Studien zeigen, dass das Interesse eine relativ große Bedeutung für die Lernerfolge aufweist. Das Ziel dieser vorliegenden empirischen Studie ist festzustellen, ob Methodenvielfalt im DaF-Unterricht das Interesse für Deutschlernen steigert. Die Ergebnisse des pädagogischen Experiments, bei dem drei Unterrichtsthemen nach Prinzipien dreier Methoden (kommunikative Methode, CLIL Methode - Content and Language Integrated Learning und CALL Methode - Computer assisted Language Learning) bearbeitet wurden, zeugen von einer größeren Senkung des Interesses in der Experimentalgruppe im Vergleich zu der Kontrollgruppe, die nur nach den Prinzipien der kommunikativen Methode Deutsch lernte. Die Resultate weisen darauf hin, dass das Interesse durch den Methodenwechsel nicht gefördert werden kann. Im Beitrag wird versucht, die Senkung des Interesses zu ergründen und anhand der Ergebnisse werden Anregungen für die Unterrichtspraxis gegeben.

Interesse - CLIL - CALL - kommunikativer Ansatz - Deutsch als Fremdsprache

\section{Einleitung}

Die Globalisierung durch Medien, Auslandsaufenthalte, Privatreisen, Studium im Ausland sowie wirtschaftliche Kontakte stellen dem slowenischen Lernenden eine Bestätigung für den großen Bedarf nach Englischkenntnissen dar. Englisch wurde zu lingua franca, zur Verkehrssprache der heutigen Welt und das Interesse der Lernenden für das Lernen des Englischen wird stark dadurch geprägt. Folglich sind die anderen meist verbreiteten Fremdsprachen in Slowenien in eine ungünstige Position gestellt. Noch vor knapp 20 Jahren lernten viele slowenische Schüler Deutsch als erste Fremdsprache, heute nur noch ganz wenige. Da auch die meisten deutschen Firmen in Slowenien Englisch als Arbeitssprache eingeführt haben, ist es nicht ganz einfach Schüler und Schülerinnen für das Erlernen der deutschen Sprache zu motivieren. Obwohl die Statistiken für Deutsch als Fremdsprache momentan eine positive 
Tendenzsteigerung erweisen ${ }^{1}$, stellen sich die Fragen, welche Faktoren das Interesse für das Deutschlernen in Slowenien beeinflussen und wie das Interesse für das Deutschlernen gesteigert werden kann. Laut Krapp (1998: 185) ist das Interesse die Hauptkomponente des Lehr-/Lerngeschehens und es verdient eine ganz besondere Aufmerksamkeit. Der moderne Fremdsprachenunterricht wird stark durch den kommunikativen Ansatz bzw. Methode ${ }^{2}$ und den Europäischen Referenzrahmen für Sprachen (Europarat 2001) geprägt. Als Hauptziel des Fremdsprachenlernens wird die Kommunikation in der Fremdsprache gestellt. Dem slowenischen Lehrplan für Deutsch in Gymnasien (Holc et al. 2008) nach sollte die kommunikative Methode im Unterricht realisiert werden. Gleichzeitig bahnen sich in der Praxis auch andere Methoden ihren Weg. Eine Hochkonjunktur erlebt vor allem die CLIL-Methode (Engl. Content and integrated language learning), die dem Grundprinzip unterliegt, Inhalte aus dem Sachfachunterricht in einer Fremdsprache zu lernen (vgl. Wolff 2009). Den Eingang in die Schule fand auch die CALL-Methode (Engl. Computer assisted language learning), denn immer häufiger werden Computerprogramme, virtuelle Lernplattformen und mobile Endgeräte im DaF-Unterricht eingesetzt. Um festzustellen ob verschiedene Methoden eine Auswirkung auf das Interesse für das Deutschlernen haben, wurde eine Untersuchung angelegt und durchgeführt, die den Einfluss von drei unterschiedlichen Methoden dreier verschiedenen Methoden im Unterricht auf das Interesse fürs Deutschlernen im slowenischen Kontext durchleuchten soll.

\subsection{Verbindung zwischen dem Interesse und den Methoden}

In der einschlägigen Literatur wird das Interesse am Fremdsprachenlernen häufig mit Motivation gleichgesetzt (vgl. Riemer 2010, Schiefele 2008, Solmecke 1983). ${ }^{3}$ Obwohl das Motivationskonstrukt in der Theorie viele Kontroversen aufweist, sind sich die Forscher einig, dass es sich es um ein multidimensionales Phänomen handelt (vgl. Dörnyei 2012, Riemer 2010, Kleppin 2002, 2001) Einige der bekanntesten Motivationsmodelle stammen von Gardner (1960, 2001), Deci und Ryan (1993), Schumann (1986) und Dörnyei (1994, 2001). Im Rahmen dieser Studie wird nur auf das Modell von Dörnyei (1994, 2001) näher eingegangen, da es für die vorliegende Studie relevant ist. Dörnyei befasste sich vor allem mit der Motivation für das schulische Fremdsprachenlernen. Seiner Meinung nach ist diese Motivation anders als die Motivation für Fremdsprachen im bilingualen Kontext. Er

1 Genauere Statistiken erhältlich unter: http://www.auswaertiges-amt.de/cae/servlet/contentblob/ 364458/publicationFile/204418/PublStatistik.pdf (Zugriffsdatum: 12. 2. 2016)

2 Aus Gründen der besseren Lesbarkeit wird auf die gleichzeitige Verwendung von Termini Ansatz und Methode verzichtet. Verwendet wird der Ausdruck Methode.

3 Da die Distinktion zwischen Motivation und Interesse auf der theoretischen Ebene für die Lehrkräfte nicht entscheidend ist, wird zwischen beiden Begriffen nicht unterschieden. Die Begriffe Interesse und Motivation werden austauchbar verwendet. 
stellte zuerst einen Klassifikationsrahmen dar, in dem zahlreiche Parameter in drei Ebenen (Sprache, Lerner, Lernsituation) klassifiziert werden (Dörnyei 1994). Später erweiterte er sein dynamisches Motivationsmodell mit sieben Dimensionen: die affektive/integrative Dimension, die instrumentelle/pragmatische Dimension, die auf den Makro-Kontext bezogene Dimension, die auf das Selbstkonzept bezogene Dimension, die auf das Ziel bezogene Dimension, die auf den erzieherischen Kontext bezogene Dimension und die auf andere Personen von Bedeutung bezogene Dimension (Dörnyei 1998, 2001). Laut Dörnyei (2001) spielen beim Fremdsprachenlernen drei Attitüden eine wichtige Rolle: Attitüde gegenüber der Gesellschaft der verwendeten Zielsprache und deren Volk, Attitüde gegenüber der Zielsprache und Attitüde gegenüber dem Fremdsprachenlernen im Allgemeinen. Diese Feststellung lässt sich gut auf das Deutschlernen im slowenischen Kontext übertragen, deswegen wird das Modell von Dörnyei (2001) im Rahmen dieser Studie übernommen und ein wenig erweitert.

Die deutsche Sprache hat in Slowenien zwar eine lange schulische Tradition, wird aber in den letzten Jahren fast ausschließlich als zweite Fremdsprache gelernt. Die Einstellung gegenüber der deutschen Sprache wird auch durch die geschichtlichen Ereignisse stark geprägt. Die Lernenden kommen in Kontakt mit der deutschen Sprache fast nur im Deutschunterricht. Folglich wird das Interessenkonstrukt mit folgenden Faktoren bestimmt:

1) persönliche Einstellung gegenüber dem Land der Zielsprache und seinem Volk,

2) persönliche Einstellung gegenüber dem Fremdsprachenlernen im Allgemeinen,

3) Einstellung gegenüber dem Deutschlernen im schulischen Kontext,

4) Einstellung gegenüber der Kontaktpflege,

5) Einstellungen der Eltern gegenüber der Zielsprache,

6) Bedeutung der Zielsprache für die Ausbildung und den Alltag.

Zahlreiche Studien, die English als Fremdsprache erforschen, weisen darauf hin, dass die Lernmotivation durch den Einsatz von bestimmten Methoden gesteigert werden kann. Lasagabaster (2011) stellte für den spanischen Kontext fest, dass Lernende, die durch CLIL Englisch lernten, wesentlich motivierter waren, als Lernende, die Englisch im klassischen Fremdsprachenunterricht lernten. Fernández Fontecha (2014) untersuchte die Motivation für Vokabellernen durch den Einsatz von CLIL und dem Englischen als Fremdsprachenunterricht und bestätigte signifikante Unterschiede nur im Primarbereich, wogegen Unterschiede im Sekundarbereich nicht signifikant waren. Lasagabaster und Lopez Beloqi (2015) verglichen den Einfluss von CLIL und dem klassischen Englischunterricht und stellten fest, dass eine positive Auswirkung von CLIL nur bei der intrinsischen und integrativen Motivation abzusehen war, bei anderen Faktoren war aber die Signifikanzwahrscheinlichkeit nicht bestimmt. 
Auch für den computerunterstützten Unterricht gibt es zahlreiche Studien (z. B. Warschauer 1996, Golonka et. al. 2014), die den positiven Einfluss auf die Motivation für Fremdsprachenlernen feststellen. Alm (2007) betont, dass motivierend vor allem sinnvoll vorbereitete Aufgaben wirken, welche von dem Lernenden als authentisches Sprachinput wahrgenommen werden. In den meisten Studien werden Ergebnisse der Probanden verglichen, die Englisch nach einer oder der anderen Methode lernen. Die vorliegende Studie versucht eine Antwort darauf zu finden, ob das Interesse für das Deutschlernen gesteigert werden kann, wenn der DaF-Unterricht nach Prinzipien mehrerer Methoden aufgebaut wird.

\section{Empirischer Teil}

\subsection{Forschungsdesign}

Der Einfluss verschiedener Methoden auf das Interesse am Deutschlernen im DaF-Unterricht wurde bisher nur spärlich beobachtet und beurteilt. Um diese Forschungslücke ein wenig zu schließen, geht diese Studie der Frage nach, ob der DaF-Unterricht, der nach Prinzipien verschiedener Methoden aufgebaut wird, auf das Interesse am Deutschlernen eine Wirkung ausübt. Ausgewählt wurden drei für den slowenischen Kontext relevante Methoden: kommunikative Methode, CLIL Methode (Engl. Content and Language Integrated Learning) und CALL Methode (Engl. Computer assisted Language Learning). Um auf die Forschungsfrage „Inwiefern verändert sich das Interesse am Deutschlernen, wenn Unterrichtsthemen im DaFUnterricht nach Prinzipien verschiedener Methoden behandelt werden?" eine Antwort zu bekommen, wurde ein pädagogisches Experiment angelegt. Das Interesse für das Deutschlernen wurde mithilfe eines Fragebogens vor und nach dem Experiment gemessen.

Die folgende Hypothese wurde festgelegt und überprüft:

- Das Interesse am Deutschlernen wird in der Experimentalgruppe wegen des Experimentfaktors (drei verschiedene Methoden: kommunikative Methode, CALL, CLIL) im Vergleich zu der Kontrollgruppe (kommunikative Methode) zunehmen.

Die Abbildung 1 zeigt den Experimentverlauf. Die erste Datenerhebung erfolgte vor dem Experiment durch den Interessenfragebogen. Probanden wurden zufällig in zwei Gruppen aufgeteilt. In der Experimentalgruppe wurden drei Methoden nacheinander eingeführt. In je 20 Stunden wurde das Thema Ich und die anderen nach den Prinzipien der kommunikativen Methode unterrichtet, das Thema Schule und Gegenstände rund um uns wurde nach den Prinzipien der CALL Methode bearbeitet und das Thema Essen und Trinken nach den Prinzipien der CLIL Methode. In der Kontrollgruppe wurden die gleichen Themen mit Prinzipien der kommunikativen 
Methode durchgenommen. Die erwähnten Themen wurden mithilfe von den ersten drei Lektionen aus dem Lehrwerk Themen aktuell 1 bearbeitet. Für die Bearbeitung des ersten Themas wurden in der Experimentalgruppe Lernmaterialien aus dem Lehrwerk Themen aktuell 1 (Lektion 1) verwendet, für das Thema Schule und Gegenstände rund um uns wurden eigene Lernmaterialien in einer MoodleLernplattform erstellt und für das Thema Essen und Trinken wurden eigene fächerübergreifende Lernmaterialien erstellt (Retelj 2014). Nach dem Experiment wurden die Daten mit demselben Fragebogen erneut erhoben.

\begin{tabular}{|c|c|c|c|}
\hline \multicolumn{4}{|l|}{ Erste Datenerhebung } \\
\hline $\begin{array}{l}\text { Experimentalgruppe } \\
(\mathrm{EG})\end{array}$ & $\begin{array}{l}\text { Kontrollgruppe } \\
(\mathrm{KG})\end{array}$ & Themen & Stundenzahl \\
\hline \begin{tabular}{|l|} 
Kommunikative \\
Methode
\end{tabular} & $\begin{array}{l}\text { Kommunikative } \\
\text { Methode }\end{array}$ & $\begin{array}{l}\text { Thema 1: Ich und die } \\
\text { anderen }\end{array}$ & 20 \\
\hline CALL Methode & $\begin{array}{l}\text { Kommunikative } \\
\text { Methode }\end{array}$ & $\begin{array}{l}\text { Thema 2: Schule und } \\
\text { Gegenstände rund um } \\
\text { uns }\end{array}$ & 20 \\
\hline CLIL Methode & $\begin{array}{l}\text { Kommunikative } \\
\text { Methode }\end{array}$ & $\begin{array}{l}\text { Thema 3: Essen und } \\
\text { Trinken }\end{array}$ & 20 \\
\hline
\end{tabular}

Abbildung 1: Verlauf des Experiments

\subsection{Methode}

\subsubsection{Forschungsteilnehmer}

An der Studie nahmen insgesamt 129 DaF-Lernende von zwei slowenischen Gymnasien teil. Zum Zeitpunkt der Untersuchung lag das Durchschnittsalter der Versuchsteilnehmer bei 15 Jahren. Die Experimentalgruppe (weiter EG) bestand aus 69 und die Kontrollgruppe (weiter KG) aus 60 Teilnehmern. In beiden Gruppen war der Teil der weiblichen Probanden größer (EG - 68,1\%, KG - 68,3\%), was auch die reale Situation an slowenischen Gymnasien widerspiegelt.

\subsubsection{Messinstrument}

Zur Datenerhebung wurde ein Interessenfragebogen in der slowenischen Sprache entwickelt (siehe Anhang). Bei der Entwicklung des Messinstruments lehnten wir uns an die bereits existierenden Fragebögen zur Motivation im Unterricht von Dörnyei ${ }^{1}$ und übernahmen die meisten Items. Um alle Faktoren aus unserem Interessenkonstrukt einzubringen, wurde das Instrument mit weiteren Items

1 Auf der persönlichen Webseite von Dörnyei sind alle Fragebögen zugänglich: http://www. zoltandornyei.co.uk/\#untitled51 (Zugriffsdatum 12. 2. 2016). 
ergänzt. Der Interessenfragebogen besteht aus 66 Aussagen - Items, die auf einer 5-stufigen Likert-Skala abgefragt wurden (1- stimmt gar nicht - 5 stimmt völlig). Der von uns erstellte Fragebogen erwies eine genügende Zuverlässigkeit. ${ }^{1}$

\subsubsection{Datenerhebung}

Die Datenerhebung fand im Schuljahr 2013/2014 statt. Der Fragebogen wurde von den Teilnehmern vor der Ausführung des Experiments im September 2013 ausgefüllt und dann noch im März 2014 nach dem Experiment. Die Resultate wurden zunächst in Excel übertragen und mit dem Programm SPSS bearbeitet. Folgende statistische Verfahren wurden eigensetzt:

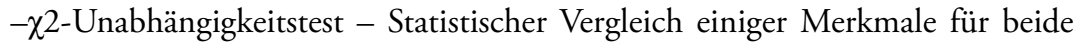
Gruppen (Geschlecht, Vorkenntnisse, Kontakte mit der deutschen Sprache außerhalb des regulären Unterrichts)

- T-Test für unabhängige Stichproben - Vergleich der Mittelwerte für zwei unabhängige Stichproben

- T-Test für abhängige Stichproben - Vergleich der Mittelwerte für zwei unabhängige Stichproben

\section{Ergebnisse und Diskussion}

Um potenzielle Unterschiede nach dem Experiment dem Experimentalfaktor zuzuschreiben, mussten vor dem Experiment einige Variablen (Geschlecht, Vorkenntnisse, Kontakte mit der deutschen Sprache außerhalb des regulären Unterrichts $^{2}$ ) kontrolliert werden. Mit dem $\chi 2$-Unabhängigkeitstest wurden keine signifikanten Unterschiede für Vorkenntnisse $(\chi 2=2,202 ; \mathrm{P}=0,179)$, Kontakte $(\chi 2=1,209 ; \mathrm{P}=0,337)$ und Geschlecht $(\chi 2=0,001, \mathrm{P}=0,979)$ bestätigt. ${ }^{3}$ Das heißt, dass die beiden Versuchsgruppen miteinander vergleichbar waren.

1 Um die Zuverlässigkeit zu überprüfen wurde der Koeffizient Cronbach alpha vor dem Experiment und nach dem Experiment für beide Gruppen berechnet. Cronbach alfa vor dem Experiment beträgt für $\mathrm{EG}=0,917$ und für $\mathrm{KG}=0,946$ und nach dem Experiment für $\mathrm{EG}=0,940$ und für $\mathrm{KG}=0,915$.

2 Einige Probanden hatten Deutschvorkenntnisse aus der Grundschule, einige kamen ins Gymnasium ohne Vorkenntnisse. In beiden Versuchsgruppen gab es überwiegend Versuchsteilnehmer mit Vorkenntnissen in der deutschen Sprache (EG - 75,4\%, KG - 63,3\%). Da Kontakte mit der deutschen Sprache außerhalb des regulären Unterrichts (Verwandte, Freunde, Fernsehen, Internet, Auslandsreise) eine wichtige Rolle zur Einstellung gegenüber der deutschen Sprache beitragen könnten, wurde auch dieser Faktor wurde beachtet. In beiden Versuchsgruppen gab es überwiegend jene Teilnehmer, welche keine Kontakte mit der deutschen Sprache außerhalb des Unterrichts pflegen (EG - 73,9\%, KG - 65,0\%).

3 Diese Untersuchung ist nur ein Teil eines größeren Dissertationsprojekts, bei dem mehrere Messinstrumente entwickelt und viele andere Daten erhoben wurden. Es wird nur auf die Daten zurückgegriffen, die für diesen Teil relevant sind. 


\subsection{Das Interesse für Deutschlernen vor und nach dem Experiment}

Analysiert wurde:

- das Interesse für einzelne Interessenkategorie,

- das Gesamtinteresse, berechnet als Resultat des ganzen Fragebogens.

Einzelne Items wurden zuerst 6 Variablen - Interessenkategorien zugeordnet: Interesse für Deutschland und Deutsche, Interesse für Fremdsprachen, Interesse für Deutsch als Schulfach, Interesse für Kontakte mit der deutschen Sprache, Interesse der Eltern für Deutsch, Bedeutung der deutschen Sprache für Ausbildung und Leben. ${ }^{1}$ Mittelwerte für einzelne Interessenkategorien wurden berechnet und miteinander verglichen.

\begin{tabular}{|l|l|l|}
\hline Interessenkategorien vor dem Experiment & EG & KG \\
\cline { 2 - 3 } & MT & MT \\
\hline Interesse für Deutschland und Deutsche & 3,61 & 3,10 \\
\hline Interesse für Fremdsprachen & 4,44 & 4,12 \\
\hline Interesse für Deutsch als Schulfach & 4,12 & 3,50 \\
\hline Interesse für Kontakte mit der deutschen Sprache & 3,51 & 3,20 \\
\hline Interesse der Eltern für Deutsch & 3,69 & 3,60 \\
\hline Bedeutung der deutschen Sprache für Ausbildung und Leben & 3,91 & 3,40 \\
\hline Gesamt & 3,91 & 3,45 \\
\hline
\end{tabular}

Tabelle 1 Mittelwerte für Interessenkategorien für beide Versuchsgruppen vor dem Experiment (EG=Experimentalgruppe, $\mathrm{KG}=$ Kontrollgruppe, $\mathrm{MT}=$ Mittelwert)

Die Analyse führte zu Ergebnissen, die bestätigen, dass vor dem Experiment die Mittelwerte für Interessenkategorien in der Experimentalgruppe höher waren als in der Kontrollgruppe. Dieser Unterschied musste statistisch überprüft werden. Dafür wurde der T-Test für unabhängige Stichproben ausgewählt. Die Unterschiede bei den Kategorien Interesse für Deutschland und Deutsche $(\mathrm{t}=4,428, \mathrm{p}=0,000)$, Interesse für Fremdsprachen $(\mathrm{t}=3,338, \mathrm{p}=0,000)$, Interesse für Deutsch als Schulfach $(\mathrm{t}=6,467, \mathrm{p}=0,000)$, Interesse für Kontakte mit der deutschen Sprache $(\mathrm{t}=2,832$, $\mathrm{p}=0,005)$, Bedeutung der deutschen Sprache für Ausbildung und Leben $(\mathrm{t}=4,754$, $\mathrm{p}=0,000)$ war statistisch signifikant. Bei der Interessenkategorie Interesse der Eltern

1 Die Nummerierung der einzelnen Items entspricht der Nummerierung im Fragebogen. In die Interessenkategorie Interesse für Deutschland und Deutsche gehören Items unter Nummern 1, 4, 13, 20, 25, 30, 31, 32, 37, 40, 53. Die Kategorie Fremdsprachen bilden Items 11, 12, 14, 22, 35, 63. Items 2, 6, 7, 17, 19, 21, 23, 24, 28, 29, 34, 39, 41, 42, 48, 49, 50, 54, 56, 60, 61, 64 werden der Kategorie Interesse für Deutsch als Schulfach zugeordnet. In die Kategorie Interesse für Kontakte mit der deutschen Sprache gehören Items 5, 44, 45, 55, 59, 65, 66. Die Kategorie Interesse der Eltern für Deutsch hat Items unter Nummern 27, 33, 36, 38, 43, 46, 47, 62. Die Kategorie Bedeutung der deutschen Sprache für Ausbildung und Leben wird mit Items 3, 8, 9, 10, 15, 16, 18 , $26,51,52,57,58$ gebildet. 
für Deutsch ( $\mathrm{t}=0,678, \mathrm{P}=0,504)$ war der Unterschied nicht statisch signifikant. Das Interesse in der Experimentalgruppe war vor dem Experiment statistisch signifikant höher als in der Kontrollgruppe. Diese Umstände mussten bei der Interpretation der Resultate im Auge behalten werden.

In der Tabelle 2 werden die Mittelwerte für Interessenkategorien nach dem Experiment ausgeführt.

\begin{tabular}{|l|l|l|}
\hline Interessenkategorien nach dem Experiment & EG & KG \\
\cline { 2 - 3 } & MT & MT \\
\hline Interesse für Deutschland und Deutsche & 3,43 & 3,24 \\
\hline Interesse für Fremdsprachen & 4,21 & 3,65 \\
\hline Interesse für Deutsch als Schulfach & 3,79 & 3,22 \\
\hline Interesse für Kontakte mit der deutschen Sprache & 3,38 & 2,96 \\
\hline Interesse der Eltern für Deutsch & 3,40 & 2,97 \\
\hline Bedeutung der deutschen Sprache für Ausbildung und Leben & 3,71 & 3,44 \\
\hline Gesamt & 3,66 & 3,24 \\
\hline
\end{tabular}

Tabelle 2 Mittelwerte für Interessenkategorien nach dem Experiment für beide Versuchsgruppen ( $\mathrm{EG}=$ Experimentalgruppe, $\mathrm{KG}=$ Kontrollgruppe, $M T-M i t t e l w e r t e)$

Die Resultate weisen darauf hin, dass das Interesse in der Experimentalgruppe (EG) nach dem Experiment größer ist als in der Kontrollgruppe (EG). Dabei darf nicht vergessen werden, dass das Interesse in der Experimentalgruppe schon vor dem Experiment größer war. Gleichzeitig ist aus der Tabelle 2 ersichtlich, dass das Interesse für Deutsch nach dem Experiment in fast allen Interessenkategorien in beiden Versuchsgruppen niedrigere Werte belegt als vor dem Experiment (siehe Tabelle 1).

Um die Unterschiede nach dem Experiment festzustellen, wurden beide Gruppen in den Interessenkategorien erneut verglichen. Dafür wurde der T-Test für unabhängige Stichproben ausgewählt. Die Signifikanz wird bei Kategorien Interesse für Fremdsprachen $(\mathrm{t}=4,325, \mathrm{p}=0,000)$, Interesse für Deutsch als Schulfach $(\mathrm{t}=4,957$, $\mathrm{p}=0,000)$, Interesse für Kontakte mit deutschen Sprache $(\mathrm{t}=3,618, \mathrm{p}=0,000)$, Interesse der Eltern für Deutsch $(\mathrm{t}=3,258, \mathrm{p}=0,001)$, Bedeutung der deutschen Sprache für Ausbildung und Leben $(\mathrm{t}=2,529, \mathrm{p}=0,013)$ bestätigt. Die Kategorie Interesse für Deutschland und Deutsche $(\mathrm{t}=1,717, \mathrm{p}=0,059)$ zeigt keine statistische Signifikanz, was bedeutet, dass die Unterschiede zwischen den beiden Versuchsgruppen auch nach dem Experiment existierten.

In der Tabelle 3 sind die Mittelwerte (MT) für einzelne Items für beide Versuchsgruppen vor und nach dem Experiment aufgelistet. Die Ergebnisse zeigen, dass die Mittelwerte der Experimentalgruppe (EG) bei den meisten Items vor und nach dem Experiment höher sind als die Mittelwerte der Kontrollgruppe 
(KG). Der Vergleich der Mittelwerte für die Experimentalgruppe vor und nach dem Experiment und der Vergleich der Mittelwerte für die Kontrollgruppe vor und nach dem Experiment, deuten aber auf eine Senkung der Mittelwerte bei der Experimentalgruppe. Die Mittelwerte in der Experimentalgruppe nach dem Experiment sind also niedriger als vor dem Experiment.

\begin{tabular}{|c|c|c|c|c|c|c|}
\hline \multirow{3}{*}{ 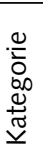 } & \multirow[t]{3}{*}{ Nr. } & \multirow[t]{3}{*}{ Item } & \multicolumn{2}{|c|}{\begin{tabular}{|l|} 
Vor dem \\
Experiment
\end{tabular}} & \multicolumn{2}{|c|}{$\begin{array}{l}\text { Nach dem } \\
\text { Experiment }\end{array}$} \\
\hline & & & EG & KG & EG & KG \\
\hline & & & MT & MT & MT & MT \\
\hline \multirow[t]{12}{*}{1} & 1. & $\begin{array}{l}\text { Ich möchte mehr deutsche Muttersprachler kennen } \\
\text { lernen. }\end{array}$ & 4,04 & 3,37 & 3,94 & 3,55 \\
\hline & 4. & $\begin{array}{l}\text { Es wäre schade, wenn Slowenien keine Kontakte mit } \\
\text { deutschsprachigen Ländern hätte. }\end{array}$ & 4,06 & 3,90 & 3,93 & 3,73 \\
\hline & 13. & $\begin{array}{l}\text { Je mehr ich deutschsprachige Menschen kenne, desto } \\
\text { mehr mag ich sie. }\end{array}$ & 3,70 & 2,93 & 3,67 & 3,52 \\
\hline & 20. & $\begin{array}{l}\text { Es freut mich, deutschsprachige Menschen } \\
\text { kennenzulernen. }\end{array}$ & 4,04 & 3,42 & 3,67 & 3,22 \\
\hline & 25. & Man kann Muttersprachlern vertrauen. & 3,00 & 2,60 & 2,96 & 3,22 \\
\hline & 30. & $\begin{array}{l}\text { Ich möchte so gut Deutsch sprechen wie die } \\
\text { Muttersprachler. }\end{array}$ & 4,04 & 3,57 & 3,91 & 3,60 \\
\hline & 31. & $\begin{array}{l}\text { Deutschkenntnisse sind für die wirtschaftliche } \\
\text { Entwicklung Sloweniens wichtig. }\end{array}$ & 3,64 & 3,15 & 3,28 & 3,10 \\
\hline & 32. & Mit Deutschen kann man leicht Kontakte anknüpfen. & 3,28 & 2,80 & 2,80 & 3,03 \\
\hline & 37. & $\begin{array}{l}\text { Ich hätte gern viele Freunde aus deutschsprachigen } \\
\text { Ländern. }\end{array}$ & 3,78 & 2,92 & 3,36 & 2,73 \\
\hline & 40. & $\begin{array}{l}\text { Deutschkenntnisse helfen mir, Deutsche besser zu } \\
\text { verstehen. }\end{array}$ & 2,72 & 2,43 & 2,68 & 2,55 \\
\hline & 53. & Deutsche sind meistens gesellig und freundlich. & 3,43 & 3,03 & 3,51 & 3,37 \\
\hline & & Gesamt & 3,61 & 3,10 & 3,43 & 3,24 \\
\hline \multirow[t]{7}{*}{2} & 11. & $\begin{array}{l}\text { Wenn ich in ein anderes Land umziehen würde, würde } \\
\text { ich seine Sprache lernen. }\end{array}$ & 4,43 & 4,15 & 4,26 & 3,62 \\
\hline & 12. & Ich möchte viele Fremdsprachen beherrschen. & 4,38 & 3,98 & 4,30 & 3,37 \\
\hline & 14. & $\begin{array}{l}\text { Ich möchte viele Fremdsprachen auf einem hohen } \\
\text { Niveau sprechen. }\end{array}$ & 4,75 & 4,38 & 4,57 & 3,63 \\
\hline & 22. & $\begin{array}{l}\text { Ich habe großes Interesse an } \\
\text { Fremdsprachenkenntnissen. }\end{array}$ & 4,68 & 4,35 & 4,42 & 4,15 \\
\hline & 35. & Ich möchte fließend Deutsch sprechen. & 4,57 & 4,23 & 4,25 & 3,60 \\
\hline & 63. & Die meisten Fremdsprachen klingen nicht komisch. & 3,86 & 3,63 & 3,46 & 3,55 \\
\hline & & Gesamt & 4,44 & 4,12 & 4,21 & 3,65 \\
\hline
\end{tabular}




\begin{tabular}{|c|c|c|c|c|c|c|}
\hline \multirow[t]{23}{*}{3} & 2. & \multirow{2}{*}{$\begin{array}{l}\text { Beim Deutschlernen mag ich alles. } \\
\text { Ich mache meine Hausaufgabe sofort, wenn ich kann. } \\
\text { (M) }\end{array}$} & \multirow{2}{*}{3,68} & \multirow{2}{*}{$\begin{array}{l}2,90 \\
3,60\end{array}$} & \multirow{2}{*}{3,61} & \multirow{2}{*}{$\begin{array}{l}3,02 \\
3,23\end{array}$} \\
\hline & 6. & & & & & \\
\hline & 7. & Deutschlernen ist keine Zeitverschwendung. $(M)$ & 4,75 & 4,12 & 4,42 & 3,65 \\
\hline & 17. & Hätte ich mehr Zeit, würde ich mehr Deutsch lernen. & 3,78 & 2,83 & 3,72 & 3,52 \\
\hline & 19. & Grunddeutschkenntnisse reichen mir nicht aus. & 4,58 & 4,10 & 4,12 & 3,48 \\
\hline & 21. & Ich gebe zu, ich lerne gern Deutsch. & 4,14 & 3,42 & 3,74 & 3,47 \\
\hline & 23. & $\begin{array}{l}\text { Ich habe vom Tag zu Tag den größeren Wunsch, } \\
\text { besser Deutsch zu beherrschen. }\end{array}$ & 4,74 & 4,12 & 4,23 & 3,00 \\
\hline & 24. & Oft lerne ich Deutsch gern in der Schule. & 4,32 & 3,37 & 3,74 & 3,73 \\
\hline & 28. & Deutsch als Schulfach gefällt mir. (M). & 4,43 & 3,90 & 4,09 & 3,40 \\
\hline & 29. & Ich möchte so viel wie möglich Deutsch lernen. & 4,12 & 3,70 & 4,04 & 3,20 \\
\hline & 34. & $\begin{array}{l}\text { Wenn ich Deutsch lernen konzentriere ich mich und } \\
\text { versuche mein Bestes zu geben. }\end{array}$ & 4,29 & 3,78 & 4,04 & 3,25 \\
\hline & 39. & Ich lerne Deutsch fast jeden Tag. & 3,01 & 2,43 & 2,41 & 2,38 \\
\hline & 41. & $\begin{array}{l}\text { Wenn ich etwas nicht verstehe, frage ich meinen/ } \\
\text { meine Deutschlehrer/Deutschlehrerin. }\end{array}$ & 3,58 & 3,20 & 3,49 & 3,03 \\
\hline & 42. & Beim Deutschlernen gebe ich mir viel Mühe. & 4,00 & 3,50 & 3,65 & 2,70 \\
\hline & 48. & Deutsch muss ein Pflichtfach in der Schule sein. & 4,25 & 3,53 & 4,04 & 3,90 \\
\hline & 49. & Deutschlernen macht Spaß. & 4,17 & 3,37 & 3,80 & 2,98 \\
\hline & 50. & $\begin{array}{l}\text { Ich habe keine Probleme komplizierte Strukturen in } \\
\text { der deutschen Sprache zu lernen. }\end{array}$ & 3,36 & 2,63 & 3,23 & 2,35 \\
\hline & 54. & $\begin{array}{l}\text { Ich sehe mir die Korrekturen meines Deutschlehrers } \\
\text { immer aufmerksam an. }\end{array}$ & 4,70 & 4,07 & 4,42 & 3,65 \\
\hline & 56. & Meine Fehler in Klassenarbeiten interessieren mich. & 4,58 & 4,22 & 4,25 & 3,55 \\
\hline & 60. & Ich lerne Deutsch wirklich gern. & 3,84 & 3,10 & 3,57 & 2,90 \\
\hline & 61. & $\begin{array}{l}\text { Auch wenn ich meine/n Lehrerin/Lehrer nicht } \\
\text { verstehe, versuche ich mich zu konzentrieren. }\end{array}$ & 4,20 & 3,57 & 3,88 & 3,60 \\
\hline & 64. & Ich habe Deutsch in der Schule wirklich gern. & 3,93 & 3,50 & 3,23 & 2,85 \\
\hline & & Gesamt & 4,12 & 3,50 & 3,79 & 3,22 \\
\hline \multirow[t]{8}{*}{4} & 5. & $\begin{array}{l}\text { Wegen meiner guten Deutschkenntnisse bin ich } \\
\text { gelassen, wenn ich Deutsch spreche. }\end{array}$ & 3,65 & 3,23 & 3,48 & 3,33 \\
\hline & 44. & $\begin{array}{l}\text { Ich möchte auch so gut Deutsch sprechen, wie die } \\
\text { anderen. }\end{array}$ & 4,36 & 3,82 & 4,16 & 3,22 \\
\hline & 45. & $\begin{array}{l}\text { Wenn ich Deutsch höre, versuche ich immer das } \\
\text { Gespräch zu verstehen }\end{array}$ & 4,41 & 3,77 & 3,88 & 2,93 \\
\hline & 55. & $\begin{array}{l}\text { Ich möchte Zeitschriften und Zeitungen auf Deutsch } \\
\text { lesen. }\end{array}$ & 4,25 & 3,65 & 4,03 & 3,07 \\
\hline & 59. & Ich mag synchronisierte Filme nicht. & 4,06 & 4,10 & 3,54 & 3,70 \\
\hline & 65. & $\begin{array}{l}\text { Ich habe viele Kontakte mit der deutschen Sprache } \\
\text { auch außerhalb der Schule. }\end{array}$ & 1,90 & 1,95 & 2,23 & 2,47 \\
\hline & 66. & $\begin{array}{l}\text { Ich sehe mir viele deutsche Filme an und höre oft } \\
\text { deutsches Radio. }\end{array}$ & 1,96 & 1,88 & 2,33 & 2,03 \\
\hline & & Gesamt & 3,51 & 3,20 & 3,38 & 2,96 \\
\hline
\end{tabular}




\begin{tabular}{|c|c|c|c|c|c|c|}
\hline \multirow[t]{9}{*}{5} & 27. & Meine Eltern helfen mir beim Deutschlernen & 2,52 & 2,55 & 2,00 & 2,02 \\
\hline & 33. & $\begin{array}{l}\text { Meine Eltern sind überzeugt, dass Deutschkenntnisse } \\
\text { für mich wichtig sind. }\end{array}$ & 4,20 & 4,17 & 4,07 & 3,72 \\
\hline & 36. & $\begin{array}{l}\text { Meine Eltern denken, dass Deutschkenntnisse im } \\
\text { Alltag brauchbar sind. }\end{array}$ & 3,90 & 3,60 & 3,59 & 3,25 \\
\hline & 38. & $\begin{array}{l}\text { Meine Eltern unterstützen mich, wenn ich etwas beim } \\
\text { Deutschunterricht nicht verstehe und meinen Lehrer } \\
\text { frage. }\end{array}$ & 4,14 & 3,73 & 3,75 & 3,18 \\
\hline & 43. & $\begin{array}{l}\text { Meine Eltern denken, dass es wichtig ist Deutsch zu } \\
\text { beherrschen }\end{array}$ & 4,23 & 4,07 & 3,90 & 2,78 \\
\hline & 46. & $\begin{array}{l}\text { Beim Deutschlernen bekomme ich viel Unterstützung } \\
\text { von meinen Eltern. }\end{array}$ & 4,41 & 4,13 & 3,88 & 3,10 \\
\hline & 47. & $\begin{array}{l}\text { Meine Eltern sind an dem Stoff beim } \\
\text { Deutschunterricht interessiert. }\end{array}$ & 3,41 & 3,32 & 3,14 & 2,82 \\
\hline & 62. & $\begin{array}{l}\text { Meine Eltern denken, ich sollte mehr Zeit der } \\
\text { deutschen Sprache widmen. }\end{array}$ & 2,72 & 3,23 & 2,84 & 2,87 \\
\hline & & Gesamt & 3,69 & 3,60 & 3,40 & 2,97 \\
\hline \multirow[t]{13}{*}{6} & 3. & $\begin{array}{l}\text { Mit Deutschkenntnissen kann ich leichter einen Job } \\
\text { finden. }\end{array}$ & 4,20 & 4,00 & 3,97 & 3,75 \\
\hline & 8. & $\begin{array}{l}\text { Deutschkenntnisse helfen mir auch auf anderen } \\
\text { Gebieten besser zu sein. }\end{array}$ & 4,04 & 3,45 & 3,51 & 3,03 \\
\hline & 9. & $\begin{array}{l}\text { Deutschkenntnisse sind wichtig, weil sie mir } \\
\text { ermöglichen andere Leute kennenzulernen und mit } \\
\text { denen zu kommunizieren. }\end{array}$ & 4,49 & 3,80 & 3,87 & 3,67 \\
\hline & 10. & Deutschkenntnisse sind heute ein Muss. & 3,61 & 2,80 & 3,35 & 3,27 \\
\hline & 15. & Deutschkenntnisse sind wichtig. $(M)$ & 4,68 & 4,25 & 4,33 & 3,57 \\
\hline & 16. & Deutschkenntnisse helfen mir beim Studium. & 4,09 & 3,62 & 4,03 & 4,10 \\
\hline & 18. & $\begin{array}{l}\text { Gute Deutschkenntnisse sind für mich ein wichtiges } \\
\text { Ziel im Leben. }\end{array}$ & 4,10 & 3,58 & 3,72 & 3,20 \\
\hline & 26. & $\begin{array}{l}\text { Das Deutschlernen ist für meine Kommunikation auf } \\
\text { Deutsch wichtig. }\end{array}$ & 4,65 & 4,33 & 4,26 & 3,90 \\
\hline & 51. & $\begin{array}{l}\text { Es wäre gut auch andere Schulfächer in der deutschen } \\
\text { Sprache zu lernen. }\end{array}$ & 2,17 & 1,72 & 2,49 & 2,28 \\
\hline & 52. & $\begin{array}{l}\text { Deutsch müssten wir schon in den niedrigen Klassen } \\
\text { der Grundschule lernen. }\end{array}$ & 3,42 & 2,93 & 3,64 & 3,37 \\
\hline & 57. & $\begin{array}{l}\text { Die deutsche Sprache ist auch für das Lernen anderer } \\
\text { Fächern geeignet. }(M)\end{array}$ & 3,48 & 2,63 & 3,32 & 3,52 \\
\hline & 58. & Deutsch ist ein wichtiges Schulfach am Gymnasium. & 4,03 & 3,72 & 4,03 & 3,58 \\
\hline & & Gesamt & 3,91 & 3,40 & 3,71 & 3,44 \\
\hline
\end{tabular}

Tabelle 3 Vergleich einzelner Items vor und nach dem Experiment für beide Versuchsgruppen ( $\mathrm{EG}=$ Experimentalgruppe, $\mathrm{KG}=$ Kontrollgruppe, $\mathrm{MT}=$ Mittelwert)

1 - Interesse für Deutschland und Deutsche, 2 - Interesse für Fremdsprachen, 3 - Interesse für Deutsch als Schulfach, 4 - Interesse für Kontakte mit der deutschen Sprache, 5 - Interesse der Eltern für Deutsch, 6 - Bedeutung der deutschen Sprache für Ausbildung und Leben; $(M)$ Positiv modifizierte Aussage 
Mit dem T-Test für unabhängige Stichproben wurden die Mittelwerte vor und nach dem Experiment ausgewertet. Die Ausführung des T-Test zeigte, dass die Unterschiede zwischen beiden Versuchsgruppen vor dem Experiment statistisch signifikant sind $(t=5,681, P=0,000)$. Auch die statistische Auswertung einzelner Items nach dem Experiment bestätigt die signifikanten Unterschiede $(t=4,629$, $\mathrm{P}=0,000)$ zwischen beiden Gruppen. Als beurteilendes Signifikanzniveau wurde 0,05 ausgewählt.

Um die Interessensteigerung oder -senkung zu beweisen, mussten die Ergebnisse von beiden Gruppen noch einmal verglichen werden.

Dafür wurde der T-Test für abhängige Stichproben für beide Versuchsgruppen durchgeführt. Zuerst werden Resultate für die Kontrollgruppe dargestellt.

\begin{tabular}{|c|c|c|c|c|c|}
\hline & & & \multirow{2}{*}{ KG } & \multicolumn{2}{|c|}{ T-Test } \\
\cline { 5 - 6 } & N & MT & Std. & $\mathbf{t}$ & $\mathbf{P}$ \\
\hline Interesse vor dem Experiment & 60 & 3,45 & 0,53 & 1,893 & 0,063 \\
\hline Interesse nach dem Experiment & 60 & 3,24 & 0,52 & & \\
\hline Vergleich & 60 & 0,21 & 0,10 & & \\
\hline
\end{tabular}

Tabelle $4 \mathrm{~T}$-Test für abhängige Stichproben für Kontrollgruppe (MT-Mittelwerte, Std-Standardabweichung)

Die Ergebnisse zeigen, dass die Unterschiede zwischen dem Interesse vor dem Experiment und dem Interesse nach dem Experiment für die Kontrollgruppe nicht signifikant sind $(\mathrm{t}=1,893, \mathrm{P}=0,063)$.

Aus der Tabelle 5 ist ersichtlich, dass die Unterschiede für die Experimentalgruppe vor und nach dem Experiment statistisch signifikant $(t=3,551, P=0,001)$ sind.

\begin{tabular}{|c|c|c|c|c|l|}
\hline & & & & \multicolumn{3}{|c|}{ T-Test } \\
EG & N & MT & Std. & t & P \\
\hline Interesse vor dem Experiment & 69 & 3,91 & 0,37 & 3,551 & 0,001 \\
\hline Interesse nach dem Experiment & 69 & 3,66 & 0,49 & & \\
\cline { 1 - 1 } Vergleich & 69 & 0,25 & 0,58 & & \\
\hline
\end{tabular}

Tabelle 5 T-Test für abhängige Stichproben für Experimentalgruppe (MT-Mittelwerte, Std-Standardabweichung)

Anhand des T-Tests für abhängige Stichproben für die Experimental- und für die Kontrollgruppe kann festgestellt werden, dass die Einführung des Experimentfaktors - drei verschiedene Methoden -, auf das Interesse eine Auswirkung hatte. Das Interesse in der Experimentalgruppe war vor und nach dem Experiment höher als in der Kontrollgruppe, der Vergleich zwischen dem Interesse vor dem Experiment 
mit dem Interesse nach dem Experiment zeigt aber eine sinkende Tendenz für die Experimentalgruppe. Die Kombination von den drei Methoden scheint demzufolge eine negative Auswirkung auf das Interesse zu haben. Die Ausgangshypothese, dass das Interesse wegen des Einsatzes verschiedener Methoden zunimmt, kann damit nicht bestätigt werden.

\section{Schlussfolgerung}

Obwohl der Einsatz verschiedener Methoden eine wichtige Rolle im Fremdsprachenunterricht spielt, lässt sich jedoch nicht generalisieren, dass der methodenvielfältige Unterricht ausschließlich zur Interessensteigerung beiträgt. Im Gegenteil, die Ergebnisse des vorliegenden Experiments führen zu der Erkenntnis, dass das Interesse sogar sinkende Tendenz aufzeigt. Hinsichtlich der Dauer des Experiments (fast sieben Monate) kann festgestellt werden, dass das Interesse in beiden Gruppen mit der Zeit sinkt. Von der zeitbedingten Abnahme des Interesses berichtet ausführlich schon Schiefele (2008). Für das sinkende Interesse könnte folgende Erklärung angeboten werden: in der Kontrollgruppe, die an einem kommunikativorientierten Unterricht teilnahm, waren die Unterschiede zwischen dem Interesse vor dem Experiment und nach dem Experiment nicht signifikant, was möglicherweise an einer Kontinuität und Vorhersehbarkeit des Lernens liegt. Lernende entwickelten ihre eigenen Lernstrategien und konnten ihr eigenes Lernen ohnehin planen. Die Experimentalgruppe dagegen befand sich in ständig wechselnden Umständen und wurde verschiedenen Unterrichtsprinzipien ausgesetzt. Lernende in der Experimentalgruppe mussten ihr eigenes Lernsystem immer wieder neu entdecken, was sowohl durch die CLIL-Methode mit fächerübergreifenden Themen als auch durch die CALL-Methode erzeugt wurde. Es ist nicht völlig auszuschließen, dass bei den Lernenden durch Prinzipien- bzw. Methodenwechsel Desinteresse verursacht wurde. In der Praxis wäre es vielleicht sinnvoller, das Augenmerk beim Unterricht auf sorgfältig durchdachte Prinzipien der einzelnen Methoden zu legen, statt ganze Unterrichtseinheiten nach Prinzipien einer Methode zu bearbeiten. Die Methodenvielfalt forderte von den Lernenden eine ständige Anpassungskraft, was vielleicht zu einer Überförderung führte, die sich als niedrigeres Interesse zeigte. An dieser Stelle muss noch hervorgehoben werden, dass die Lernenden dem Einsatz einer Methode nur für eine beschränkte Zeit ausgesetzt waren und wenig Möglichkeit hatten, deren Effizienz zu verinnerlichen. Da sie bei jeder nächsten Einheit eine andere Methode erlebten, sollte man die Möglichkeit in Erwägung ziehen, dass diese Wechselhaftigkeit zu einer immer geringeren Anpassungsfähigkeit führte, was sich am Ende als sinkendes Interesse zeigte. 


\section{Literatur}

Alm, Antonie (2007): Motivationstheoretische Grundbedingungen für den erfolgreichen Einsatz von Neuen Medien im Fremdsprachenunterricht. In: Zeitschrift für Interkulturellen Fremdsprachenunterricht 12:1, 2007, 23 S.

Deci, Edward L. / Ryan, Richard M. (1993): Die Selbstbestimmungstheorie der Motivation und ihre Bedeutung für die Pädagogik. In: Zeitschrift für Pädagogik 39/2/1993. S. $223-238$

Dörnyei, Zoltán (1994): Motivation and motivating in the foreign language classroom. In: The Modern Language Journal 78/1994. S. 273-284.

Dörnyei, Zoltán (1998): Motivation in second and foreign language learning. In: Language Teaching 31/1998. S. 117-135.

Dörnyei, Zoltán (2001): Teaching and Researching Motivation. Harlow, Longman.

Dörnyei, Zoltán (2002): Wie motiviere ich richtig? In: Fremdsprache Deutsch 26/2002. S. $16-17$.

Dörnyei, Zoltán (2007): Research methods in applied linguistics: Quantitative, qualitative and mixed methodologies. Oxford University Press, Oxford.

Dörnyei, Zoltán (2012): Motivation in language learning. Shanghai Foreign Language Education Press, Shanghai.

Europarat (2001): Gemeinsamer europäischer Referenzrahmen für Sprachen: lernen, lehren, beurteilen. Langenscheidt, Berlin.

Gardner, Richard C. (2001): Integrative motivation and second language acquisition. In: Gardner, Robert C. (1960): Motivational Variables in Second-Language Acquisition. Montreal: McGill Universität. (unveröffentlichte Doktorarbeit). Erhältlich unter: http:// publish.uwo.ca/-gardner/docs/phd.pdf (1. 9. 2015).

Golonka, Ewa. M. / Bowles, Anita. R. / Frank, Viktor. M. / Richardson, Dorna. L. / Freynik, Suzanne. (2014): Technologies for foreign language learning: a review of technology types and their effectiveness. In: Computer Assisted Language Learning 27/1 S. 70-105.

Holc Nada et al. (2008). Učni načrt. Nemščina. Gimnazija. Erhältlich unter: http://www. mss.gov.si/fileadmin/mss.gov.si/pageuploads/podrocje/ss/programi/2008/Gimnazije/ UN_NEMSCINA_gimn.pdf (12.2. 2016).

Kleppin, Karin (2001): Motivation. Nur ein Mythos? (I). In: Deutsch als Fremdsprache, Zeitschrift zur Theorie und Praxis des Deutschunterrichts für Ausländer 4/2001. S. 219-225.

Kleppin, Karin (2002): Motivation. Nur ein Mythos? (II). In: Deutsch als Fremdsprache, Zeitschrift zur Theorie und Praxis des Deutschunterrichts für Ausländer 1/2002. S. 26-30.

Krapp, Andreas (1998): Entwicklung und Förderung von Interessen im Unterricht. In: Psychologie in Erziehung und Unterricht, 45. S. 186-203.

Lasagabaster, David / Lopez Beloqi, Raquel (2015): The Impact of Type of Approach (CLIL Versus EFL) and Methodology (Book-Based Versus Project Work) on Motivation. In: Porta Linguarum 23/1/2015. S. 41-57.

Lasagabaster, David (2011): English achivement and student motivation in CLIL and ELF settings. In: Innovation in Language Learning and Teaching 5/1/2011. S. 3-18.

Retelj, Andreja (2014): Vpliv različnih didaktičnih pristopov na razvoj leksikalne zmožnosti pri pouku tujega jezika. Doktorsko delo. Univerza v Ljubljani. Filozofska fakulteta, Ljubljana.

Riemer, Claudia (2010): Motivation. In: Hallet Wolfgang / Königs Frank G. (Hg.): Handbuch Fremdsprachendidaktik. Seelze-Velber, Kallmeyer / Klett. S. 168-172.

Schiefele, Ullrich (2008): Lernmotivation und Interesse. In: Schneider Wolfgang / Hasselhorn Marcus (Hg.): Handbuch der Pädagogischen Psychologie. Göttingen, Hofrefe Verlag. S. 38-48. 
Schumann, John. H. (1986): Research on the acculturation model for second language acquisition. In: Journal of Multilingual and Multicultural Development. 7, S. 379-392.

Solmecke, G. (Hg.) (1983): Motivation und Motivieren im Fremdsprachenunterricht. Paderborn, Ferdinand Schöningh Verlag.

Warschauer, Mark (1996): Motivational aspects of using computers for writing and communication. In: Warschauer, Mark (Hg.): Telecollaboration in foreign language learning. Honolulu, HI, Second Language Teaching \& Curriculum Center (University of Hawai'i Press). S. 29-46.

Wolff, Dieter (2009): Content and language integrated learning. In: Knapp, K. / Seidlhofer, B. (Hrsg.). Handbook of Foreign Language Communication and Learning. Berlin: de Gruyter. S. 545-572. 


\section{Anhang 1}

\section{Fragebogen zum Interesse am Deutschlernen}

\section{Dragi dijak, draga dijakinja!}

V okviru doktorske raziskave, ki jo izvajam, te prosim, da rešiš vprašalnik o interesu za učenje nemščine. Vsi tvoji odgovori in osebni podatki bodo varovani in uporabljeni izključno $\mathbf{v}$ raziskovalne namene.

Hvala za sodelovanje Andreja Retelj!

Ime in priimek:

\section{Obkroži spol}

Predznanje iz osnovne šole

Število let učenja v OŠ

Stiki z nemščino izven šole
DA NE
1) 0-1 leto
2) več kot 1 leto

DA NE

\section{INTERES ZA UČENJE NEMŠČINE}

Pred teboj je 66 trditev, s katerimi ugotavljam tvoj interes za učenje nemščine. Vljudno prosim, da pozorno prebereš vsako trditev in jo oceniš na lestvici od 1 do 5 , kjer 1 pomeni popolno nestrinjanje in 5 popolno strinjanje. Pri vsakem vprašanju obkroži samo eno ustrezno številko.

$\begin{array}{llllll}1 & \text { popolnoma se ne strinjam } & 2 & 3 & 4 & 5\end{array}$




\begin{tabular}{|c|c|c|c|c|c|c|}
\hline & Trditev & \multicolumn{5}{|c|}{ Moja ocena } \\
\hline 1. & Rad bi poznal več naravnih govorcev nemščine. & 1 & 2 & 3 & 4 & 5 \\
\hline 2. & Pri učenju nemščine mi je vse všeč. & 1 & 2 & 3 & 4 & 5 \\
\hline 3. & Z znanjem nemščine bom lažje dobil službo. & 1 & 2 & 3 & 4 & 5 \\
\hline 4. & $\begin{array}{l}\text { Škoda bi bilo, če Slovenija ne bi imela stikov z nemško } \\
\text { govorečimi deželami. }\end{array}$ & 1 & 2 & 3 & 4 & 5 \\
\hline 5. & $\begin{array}{l}\text { Zaradi znanja nemščine sem bolj sproščen, če govorim z ljudmi, } \\
\text { ki govorijo nemško. }\end{array}$ & 1 & 2 & 3 & 4 & 5 \\
\hline 6. & $\begin{array}{l}\text { Z delanjem domače naloge za nemščino odlagam, kar se da } \\
\text { dolgo. }\end{array}$ & 1 & 2 & 3 & 4 & 5 \\
\hline 7. & Učenje nemščine je izguba časa. & 1 & 2 & 3 & 4 & 5 \\
\hline 8. & sčine sem lahko boljši tudi na drugih področjih. & 1 & 2 & 3 & 4 & 5 \\
\hline 9. & $\begin{array}{l}\text { Znanje nemščine je pomembno, ker mi omogoča, da spoznam } \\
\text { različne ljudi in se z njimi sporazumevam. }\end{array}$ & 1 & 2 & 3 & 4 & 5 \\
\hline 10. & Znanje nemščine je danes nujno potrebno. & 1 & 2 & 3 & 4 & 5 \\
\hline 11. & go državo, bi & 1 & 2 & 3 & 4 & 5 \\
\hline 12. & Rada & 1 & 2 & 3 & 4 & 5 \\
\hline 13. & Bolj k & 1 & 2 & 3 & 4 & 5 \\
\hline 14. & ahko veliko tujih & 1 & 2 & 3 & 4 & 5 \\
\hline 15. & emščine ni pomembno. & 1 & 2 & 3 & 4 & 5 \\
\hline 16. & Znan & 1 & 2 & 3 & 4 & 5 \\
\hline 17. & Če bi imel čas, bi s & 1 & 2 & 3 & 4 & 5 \\
\hline 18. & Znanje nemščine mi & 1 & 2 & 3 & 4 & 5 \\
\hline 19. & Nimam želje, da bi znal kaj več nemščine kot samo osnove. & 1 & 2 & 3 & 4 & 5 \\
\hline 20. & ti ljudi, ki go & 1 & 2 & 3 & 4 & 5 \\
\hline 21. & im rad. & 1 & 2 & 3 & 4 & 5 \\
\hline 22. & ne zanima. & 1 & 2 & 3 & 4 & 5 \\
\hline 23. & Imam čedalje manjše želje, da bi dol & 1 & 2 & 3 & 4 & 5 \\
\hline 24. & Včasih si želim, da se mi v šoli ne bi bilo treba učiti nem & 1 & 2 & 3 & 4 & 5 \\
\hline 25. & Maternim govorcem nemščine lahko zaupaš. & 1 & 2 & 3 & 4 & 5 \\
\hline 26. & $\begin{array}{l}\text { Učenje nemščine je pomembno, ker se bom lažje sporazumeval v } \\
\text { nemščini. }\end{array}$ & 1 & 2 & 3 & 4 & 5 \\
\hline
\end{tabular}




\begin{tabular}{|l|l|l|l|l|l|l|}
\hline 27. & Starši mi pomagajo pri učenju nemščine. & 1 & 2 & 3 & 4 & 5 \\
\hline 28. & Nemščine ne maram. & 1 & 2 & 3 & 4 & 5 \\
\hline 29. & Rad bi se naučil, kar se da veliko nemščine. & 1 & 2 & 3 & 4 & 5 \\
\hline 30. & Želim si govoriti tako dobro nemško kot naravni govorci. & 1 & 2 & 3 & 4 & 5 \\
\hline 31. & Za razvoj Slovenije je pomembno, da dobro znamo tudi nemško. & 1 & 2 & 3 & 4 & 5 \\
\hline 32. & Z Nemci je enostavno vzpostaviti kontakt. & 1 & 2 & 3 & 4 & 5 \\
\hline 33. & Moji starši menijo, da je pomembno, da se učim nemško. & 1 & 2 & 3 & 4 & 5 \\
\hline 34. & $\begin{array}{l}\text { Med učenjem nemščine se poskušam zbrati in res dobro opraviti } \\
\text { naloge. }\end{array}$ & 1 & 2 & 3 & 4 & 5 \\
\hline 35. & Želim si tekoče govoriti nemško. & 1 & 2 & 3 & 4 & 5 \\
\hline 36. & $\begin{array}{l}\text { Moji starši menijo, da je znanje nemščine pomembno tudi v } \\
\text { vsakdanjem življenju. }\end{array}$ & 1 & 2 & 3 & 4 & 5 \\
\hline 37. & Želim si, da bi imel veliko prijateljev iz nemško govorečih dežel.. & 1 & 2 & 3 & 4 & 5 \\
\hline 38. & $\begin{array}{l}\text { Starši me spodbujajo, da učitelja vprašam za pomoč, če imam pri } \\
\text { nemščini težave. }\end{array}$ & 1 & 2 & 3 & 4 & 5 \\
\hline 39. & Skoraj vsak dan se učim nemščino. & 1 & 2 & 3 & 4 & 5 \\
\hline 40. & Znanje nemščine mi pomaga razumeti, kako Nemci razmišljajo. & 1 & 2 & 3 & 4 & 5 \\
\hline 41. & $\begin{array}{l}\text { Če pri nemščini česa ne razumem, vedno vprašam učitelja za } \\
\text { pomoč. }\end{array}$ & 1 & 2 & 3 & 4 & 5 \\
\hline 42. & Pri nemščini se zelo trudim. & 1 & 2 & 3 & 4 & 5 \\
\hline 43. & Moji starši menijo, da je pomembno znati nemško. & 1 & 2 & 3 & 4 & 5 \\
\hline 44. & $\begin{array}{l}\text { Če slišim koga govoriti dobro nemško, si želim, da bi tudi jaz } \\
\text { govoril tako dobro. }\end{array}$ & 1 & 2 & 3 & 4 & 5 \\
\hline 45. & Vedno poskušam razumeti, če slišim nemški jezik. & 1 & 2 & 3 & 4 & 5 \\
\hline 46. & Starši me spodbujajo pri učenju nemščine. & 1 & 2 & 3 & 4 & 5 \\
\hline 47. & Moje starše zanima, kaj se učim pri nemščini. & 1 & 2 & 3 & 4 & 5 \\
\hline 48. & Nemščina ne bi smela biti obvezni predmet v gimnaziji. & 1 & 2 & 3 & 4 & 5 \\
\hline 49. & Učenje nemščine ni zabavno. & 1 & 2 & 3 & 4 & 5 \\
\hline 50. & Učenje bolj zapletenih struktur v nemščini me ne moti. & 2 & 3 & 4 & 5 \\
\hline & & & \\
\hline
\end{tabular}




\begin{tabular}{|l|l|l|l|l|l|l|}
\hline 51. & $\begin{array}{l}\text { Dobro bi bilo, če bi se učili v šoli kakšen drug predmet tudi v } \\
\text { nemščini. }\end{array}$ & 1 & 2 & 3 & 4 & 5 \\
\hline 52. & $\begin{array}{l}\text { Nemščino bi se morali učiti že v prvi ali drugi triadi v osnovni } \\
\text { Šoli. }\end{array}$ & 1 & 2 & 3 & 4 & 5 \\
\hline 53. & Nemci so običajno družabni in prijazni. & 1 & 2 & 3 & 4 & 5 \\
\hline 54. & Če učitelj popravi moj izdelek, si pogledam napake. & 1 & 2 & 3 & 4 & 5 \\
\hline 55. & Rad bi znal brati časopise in revije v nemščini. & 1 & 2 & 3 & 4 & 5 \\
\hline 56. & $\begin{array}{l}\text { Kadar dobim vrnjen izdelek pri nemščini, me napake ne } \\
\text { zanimajo. }\end{array}$ & 1 & 2 & 3 & 4 & 5 \\
\hline 57. & Nemščina ni primeren jezik, da bi se v njej učili druge predmete. & 1 & 2 & 3 & 4 & 5 \\
\hline 58. & Nemščina je pomemben predmet v srednji šoli. & 1 & 2 & 3 & 4 & 5 \\
\hline 59. & $\begin{array}{l}\text { Raje vidim, da so nemški filmi sinhronizirani v slovenščino, kot } \\
\text { pa da imajo podnapise. }\end{array}$ & 1 & 2 & 3 & 4 & 5 \\
\hline 60. & Res se rad učim nemščino. & 1 & 2 & 3 & 4 & 5 \\
\hline 61. & $\begin{array}{l}\text { Kadar pri pouku ne razumem, kaj učitelj v nemščini razlaga, se } \\
\text { ne koncentriram. }\end{array}$ & 1 & 2 & 3 & 4 & 5 \\
\hline 62. & Moji starši menijo, da bi se moral bolj posvečati nemščini. & 1 & 2 & 3 & 4 & 5 \\
\hline 63. & Večina tujih jezikov mi zveni nekoliko čudno. & 1 & 2 & 3 & 4 & 5 \\
\hline 64. & Nemščino imam zelo rad. & 1 & 2 & 3 & 4 & 5 \\
\hline 65. & Z nemščino imam izven šole veliko stikov. & 1 & 2 & 3 & 4 & 5 \\
\hline 66. & $\begin{array}{l}\text { Gledam veliko nemških filmov ali poslušam veliko radijskih } \\
\text { oddaj. }\end{array}$ & 1 & 2 & 3 & 4 & 5 \\
\hline
\end{tabular}

\section{Hvala za sodelovanje!}

asist. dr. Andreja Retelj

Filozofska fakulteta

Aškerčeva 2

Univerzita v Lublani

1000 Ljubljana

andreja.retelj@gmail.com 